\title{
Electron production by sensitizing gold nanoparticles irradiated by fast ions
}

\author{
Alexey V. Verkhovtsev, ${ }^{1,2, *}$ Andrei V. Korol, ${ }^{1,3, \dagger}$ and Andrey V. Solov’yov ${ }^{1,2, \ddagger}$ \\ ${ }^{1}$ MBN Research Center, Altenhöferallee 3, 60438 Frankfurt am Main, Germany \\ ${ }^{2}$ A.F. Ioffe Physical-Technical Institute, Politekhnicheskaya ul. 26, 194021 St. Petersburg, Russia \\ ${ }^{3}$ Department of Physics, St. Petersburg State Maritime Technical University, \\ Leninskii prospekt 101, 198262 St. Petersburg, Russia
}

\begin{abstract}
The yield of electrons generated by gold nanoparticles due to irradiation by fast charged projectiles is estimated. The results of calculations are compared to those obtained for pure water medium. It is demonstrated that a significant increase in the number of emitted electrons arises from collective electron excitations in the nanoparticle. The dominating enhancement mechanisms are related to the formation of (i) plasmons excited in a whole nanoparticle, and (ii) atomic giant resonances due to excitation of $d$ electrons in individual atoms. Decay of the collective electron excitations in a nanoparticle embedded in a biological medium thus represents an important mechanism of the low-energy electron production. Parameters of the utilized model approach are justified through the calculation of the photoabsorption spectra of several gold nanoparticles, performed by means of time-dependent density-functional theory.
\end{abstract}

\section{INTRODUCTION}

In this paper, we perform a theoretical and numerical analysis of electron production by gold nanoparticles irradiated by fast ions. It is demonstrated that gold nanoparticles significantly enhance the electron yield due to the collective response to an external electric field of a charged projectile. A significant increase in the number of emitted electrons comes from the two distinct types of collective electron excitations. Plasmons, i.e. collective excitations of delocalized valence electrons, dominate the spectra of electron emission from metallic nanoparticles in the energy range of about $1-10 \mathrm{eV}$. For higher electron energies (of a few tens of $\mathrm{eV}$ ), the main contribution to the electron yield arises from the atomic giant resonance associated with the collective excitation of $5 d$ electrons in individual atoms of a nanoparticle. As a result of these effects, the number of the low-energy electrons generated by the gold nanoparticle of a given size significantly exceeds that produced by an equivalent volume of water. Thus, decay of the collective electron excitations formed in the nanoparticles represents an important mechanism of generation of the low-energy electrons.

At present, a vivid scientific interest is in utilizing noble metal nanoparticles as dose enhancers in cancer treatments with ionizing radiation [1-4]. Injection of sensitizing nanoparticles into a tumor can increase relative biological effectiveness of the ionizing radiation, defined as a ratio of the doses delivered with photons and with a given charged projectile, leading to the same radiobiological effect. After the first experimental evidence of radiosensitization by gold nanoparticles [5] a number of follow-up experiments with noble metal and other metallic nanoparticles were performed in recent years [6-10].

\footnotetext{
*verkhovtsev@mail.ioffe.ru

$\dagger$ korol@th.physik.uni-frankfurt.de

‡ solovyov@mbnresearch.com
}

Biodamage due to ionizing radiation involves a number of phenomena, which happen on various spatial, time and energy scales. The key phenomena can be described within the so-called multiscale approach to the physics of radiation damage with ions [11]. In ion-beam cancer therapy [12-14], which is one of the promising modern techniques for cancer treatment, radiation damage is initiated by ions incident on tissue. Propagating through a biological medium, the projectiles deposit their kinetic energy by exciting and ionizing the medium. This interaction leads also to the production of secondary electrons as well as free radicals and other reactive oxygen species. It is currently acknowledged that all these secondary species largely cause the biological damage $[11,15-17]$. The lowenergy electrons, having the kinetic energy from a few $\mathrm{eV}$ to several tens of $\mathrm{eV}$, have been shown to act as important agents of biodamage [18-20]. In particular, it was indicated that such electrons can produce damage to biomolecules by dissociative electron attachment [21].

It is acknowledged that an important mechanism of excitation/ionization of metallic clusters and nanoparticles, as well as some other nanoscale systems, relies on the formation of plasmons, i.e. collective excitations of delocalized valence electrons that are induced by an external electric field [22, 23]. These excitations appear as prominent resonances in the excitation/ionization spectra of various systems, and the position of the resonance peak depends strongly on the type of a system. In the case of metallic clusters, a typical energy of the plasmon excitations is about several electronvolts, so the resonance peak is located in the vicinity of the ionization threshold $[24,25]$. The effects of resonant enhancement of ionization in atomic (metal, in particular) clusters exposed to a laser field were reviewed in Ref. [26, 27].

In the recent Monte Carlo simulation [28], the authors claimed to include the contribution of plasmon excitations when calculating the cross sections of electron and proton impact on noble metal nanoparticles. However, only "...the most simplest type of volume plasmon excitation..." was accounted for in those simulations [28]. 
On this basis, it was stated that the plasmon excitation does not play an important role in the process of electron emission from metallic nanoparticles, contributing much less to the overall cross sections than individual excitations.

In this paper, we demonstrate that the decay of plasmon excitations formed in gold nanoparticles is an important mechanism of generation of the low-energy secondary electrons. As we point out, the dominating contribution to the electron yield due to plasmon excitations comes from the surface plasmon, since its contribution to the ionization cross section exceeds that of the volume plasmon, considered in Ref. [28], by an order of magnitude.

Another important mechanism of low-energy electron production by sensitizing nanoparticles is associated with the collective excitation of $d$ electrons in individual atoms. These excitations result in the formation of the so-called atomic giant resonances in the ionization spectra of many-electron atoms $[29,30]$.

The results of the present calculations are compared to those carried out for pure water medium. We demonstrate that the number of low-energy electrons produced by the gold nanoparticle of a given size exceeds that produced by an equivalent volume of water by an order of magnitude. Providing this quantitative analysis, we indicate the advantage of using gold nanoparticles in cancer treatment with ionizing radiation. This result supports the conclusions of the recent experimental studies [31, 32] which revealed the importance of gold nanoparticles in facilitating the production of low-energy electrons, which are responsible for DNA damage.

The contribution of the plasmon excitations is evaluated by means of a model approach based on the plasmon resonance approximation (PRA) [22, 33-35]. Parameters of the utilized model are justified by calculating photoabsorption spectra of several small gold nanoparticles. To validate our approach, the PRA-based spectra are compared with those obtained by means of a more advanced method, namely by time-dependent densityfunctional theory (TDDFT) [36]. To evaluate the contribution of individual atomic excitations, we introduce an analytical expression, which relates the cross section of photoionization with that of inelastic scattering in the dipole approximation. In this paper, we consider gold nanoparticles as a case study but indicate that the introduced methodology is a general one and can be applied for other nanoscale systems, which are proposed as sensitizers in ion-beam cancer therapy.

The paper is organized as follows. Section II is devoted to the description of the theoretical framework. We outline the main points of the TDDFT approach and briefly overview the PRA. The results of the calculations and their analysis are presented in Section III. The photoionization spectra of gold clusters obtained by means of TDDFT are discussed in Section III A. In Section III B, we analyze the nature of the low-energy peak in the photoabsorption spectra of gold clusters and provide an ex- planation of why this feature can be attributed to a collective, plasmon-type excitation of valence electrons. In Section III C, we analyze the contribution of the plasmon excitations to the electron production by gold nanoparticles due to irradiation with fast ions. In Section III D, we account for the individual atomic excitations and give the resulting quantitative analysis for the electron yield from the gold nanoparticles. In Section III E, we consider different kinematic conditions and estimate the role of the plasmon excitations in the low-energy electron production as a function of the nanoparticle size and the projectile velocity. Finally, we draw the conclusions from this work.

The atomic system of units, $m_{e}=|e|=\hbar=1$, is used throughout the paper.

\section{THEORETICAL FRAMEWORK AND COMPUTATIONAL DETAILS}

Studying the electron production by gold nanoparticles irradiated by fast ions, we account for the two collective electron effects, namely excitation of delocalized valence electrons in a nanoparticle (plasmons) and that of $d$ electrons in individual atoms (giant resonances). These phenomena occur in various processes of interaction of ionizing radiation with matter. In particular, dipole collective excitations result in the formation of prominent resonances in the photoabsorption spectra of atomic clusters and nanoparticles [22, 23], while the impact ionization cross sections comprise also the contributions of higher multipole terms [34]. As we demonstrate below, the total photoabsorption spectrum of a gold nanoparticle in the energy region up to $60 \mathrm{eV}$ is approximately equal to the sum of the plasmon contribution and that of the $5 d$ electron excitations in individual atoms, $\sigma_{\gamma} \approx \sigma_{\mathrm{pl}}+\sigma_{5 \mathrm{~d}}$. Similar to the photoionization, the two distinct types of collective electron excitations appear in the process of impact ionization. On this basis, we provide a methodology for analyzing the role of these contributions to the electron production by sensitizing nanoparticles separately.

To evaluate the contribution of the plasmon excitations, we have utilized the PRA approach [22, 33-35]. It postulates that the dominating contribution to the cross section comes from collective electron excitations, while single-particle effects give much smaller contribution in the vicinity of the plasmon resonance [37, 38]. During the past decades, this approach has provided a clear physical explanation of the resonant-like structures in photoionization spectra [33, 39, 40] and differential inelastic scattering cross sections [38, 41-45] of sodium clusters and carbon fullerenes by the photon and electron impact. It was also applied [33, 46-48] to describe the dynamic response of alkali and noble metal clusters in the processes of radiative electron capture, polarization bremsstrahlung and multiphoton excitation.

The PRA relies on a few parameters, which include the oscillator strength of the plasmon excitation, posi- 
tion of the plasmon resonance peak and its width. The choice of these parameters can be justified by comparing the model-based cross sections either with experimental data or with the results of more advanced calculations. Ref. [31, 32] provided an experimental evidence that a considerable portion of radiosensitization by gold nanoparticles arises from the emitted low-energy electrons. The experimental yield of $0-15 \mathrm{eV}$ secondary electrons emitted from gold nanoparticles of about $5 \mathrm{~nm}$ in diameter was presented in Ref. [31]. The cited paper demonstrated an evidence of DNA damage by the lowenergy electrons produced by irradiation of the nanoparticles with fast charged projectiles. However, to the best of our knowledge, there are no experimentally measured impact ionization cross sections of gold clusters and nanoparticles with photons and charged projectiles covering the photon energy / energy loss range of about $1-10 \mathrm{eV}$. Therefore, we have calculated photoionization spectra of several gold clusters by means of TDDFT and then fitted the $a b$ initio-based spectra with those calculated within the model approach. Such a methodology allowed us to define the resonance frequencies and to calculate the oscillator strength of the plasmon excitations in gold nanoparticles. Note that values of the plasmon width cannot be obtained directly on the basis of the utilized model. A precise calculation of the widths can be performed by analyzing the decay of the collective excitation mode into the incoherent sum of single-electron excitations. This process should be considered within the quantum-mechanical framework [38] and cannot be treated within the classical physics framework, as the PRA does. Thus, the widths of the plasmon excitations were chosen to obtain the best agreement with the results of the TDDFT calculations.

\section{A. TDDFT calculations of photoabsorption spectra}

As a case study we have considered four threedimensional gold clusters consisting of 18 to 42 atoms, namely $\mathrm{Au}_{18}, \mathrm{Au}_{20}, \mathrm{Au}_{32}$, and $\mathrm{Au}_{42}$. The former two systems, which were observed experimentally, possess $C_{2 v}$ and $T_{d}$ symmetry, respectively $[49,50]$. The latter two are hollow, fullerene-like icosahedral structures whose high stability was predicted theoretically on the basis of DFT calculations [51-53].

The photoabsorption spectra have been calculated as follows. At first, we performed the geometry optimization using the Gaussian 09 package [54]. The optimization procedure was performed by means of DFT within the generalized gradient approximation (GGA) and using the effective-core potential CEP-121G basis set [55] augmented by $d$-polarization functions. The utilized basis set has the $4 f$ frozen core, so that 19 electrons $(5 s, 5 p, 5 d$, and $6 s$ ) from each gold atom were explicitly treated in the course of optimization. To account for the exchange and correlation corrections, the functional of Perdew, Burke and Ernzerhof [56] was utilized. Different spin multiplic- ities were considered in the course of geometry optimization. Figure 1 shows the ground-state geometries of the studied clusters.
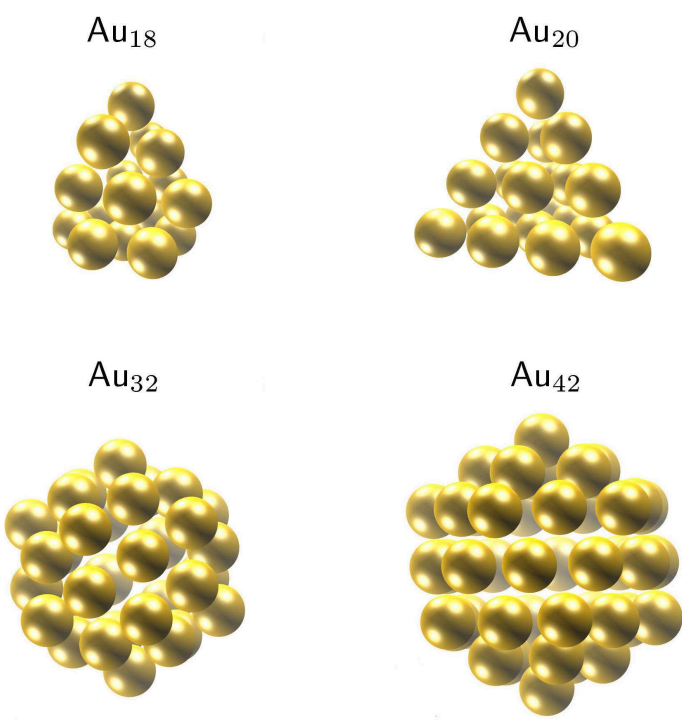

FIG. 1. Optimized structures of the studied gold clusters.

The linear response function of the clusters was calculated within the dipole approximation. Within this framework [57], the external potential acting on a system is represented as a sum of a time-independent part, $v_{\text {ext }}^{0}(\mathbf{r})$, and a time-dependent perturbation, $v_{\text {ext }}^{\prime}(\mathbf{r}, t)$. The time evolution of the electron density, $\rho(\mathbf{r}, t)$, is then represented as a sum of the unperturbed ground-state density and its variation due to $v_{\text {ext }}^{\prime}(\mathbf{r}, t)$ :

$$
\rho(\mathbf{r}, t)=\rho_{0}(\mathbf{r})+\delta \rho(\mathbf{r}, t)
$$

Performing the Fourier transform of the timedependent quantities involved, one gets the response of the system to an external perturbation in the frequency representation. For the perturbation due to a uniform electric field, $v_{\text {ext }}^{\prime}(\mathbf{r}, \omega)=-\mathbf{E}(\omega) \cdot \mathbf{r}$, the Fourier transform of the induced dipole moment reads

$$
d_{i}(\omega)=\sum_{j} \alpha_{i j}(\omega) E_{j}(\omega)
$$

Here $i, j$ denote the Cartesian components, and $\alpha_{i j}(\omega)$ is the dynamic polarizability tensor which describes the linear response of the system to the external electric field:

$$
\alpha_{i j}(\omega)=-\int r_{i} \chi\left(\mathbf{r}, \mathbf{r}^{\prime}, \omega\right) r_{j}^{\prime} \mathrm{d} \mathbf{r} \mathrm{d} \mathbf{r}^{\prime}
$$

$\chi\left(\mathbf{r}, \mathbf{r}^{\prime}, \omega\right)$ is the generalized frequency-dependent susceptibility of the system, and $r_{i}$ and $r_{j}^{\prime}$ are the components of the position vectors $\mathbf{r}$ and $\mathbf{r}^{\prime}$. The photoabsorption cross section is related to the imaginary part of the diagonal 
elements of the polarizability tensor $\alpha_{i j}(\omega)$ through

$$
\sigma(\omega)=\frac{4 \pi \omega}{3 c} \sum_{j=1}^{3} \operatorname{Im} \alpha_{j j}(\omega)
$$

where $c$ is the speed of light.

Within the approach introduced in Ref. [58-60], the electron density variation, $\delta \rho(\mathbf{r}, \omega)$, is expressed via the so-called Liouvillian operator $\mathcal{L}$,

$$
(\omega-\mathcal{L}) \cdot \delta \rho(\mathbf{r}, \omega)=\left[v_{\text {ext }}^{\prime}(\mathbf{r}, \omega), \rho_{0}\right],
$$

whose action onto $\delta \rho(\mathbf{r}, \omega)$ is defined as:

$$
\begin{aligned}
\mathcal{L} \cdot \delta \rho(\mathbf{r}, \omega) & =\left[H_{0}, \delta \rho(\mathbf{r}, \omega)\right] \\
& +\left[v_{\mathrm{H}}^{\prime}(\mathbf{r}, \omega), \rho_{0}\right]+\left[v_{\mathrm{xc}}^{\prime}(\mathbf{r}, \omega), \rho_{0}\right] .
\end{aligned}
$$

Here $H_{0}$ is the ground-state Kohn-Sham Hamiltonian calculated within the DFT approach. The quantities $v_{\mathrm{H}}^{\prime}(\mathbf{r}, \omega)$ and $v_{\mathrm{xc}}^{\prime}(\mathbf{r}, \omega)$ stand for the linear variations of the frequency-dependent electrostatic and exchangecorrelation potentials, respectively [60]. The polarizability tensor $\alpha_{i j}(\omega)$ is defined then by the off-diagonal matrix element of the resolvent of the Liouvillian $\mathcal{L}$ :

$$
\alpha_{i j}(\omega)=-\left\langle r_{i} \mid(\omega-\mathcal{L})^{-1} \cdot\left[r_{j}, \rho_{0}\right]\right\rangle
$$

which is calculated using the Lanczos recursion method (see Ref. [58-60] for details). Based on the frequency representation of the response function, this method allows one to calculate the total photoabsorption spectrum of a complex many-electron system in a broad energy range without repeating time-consuming operations for different excitation energies [58].

The photoabsorption spectra of the clusters were obtained using the TDDFPT module [61] of the Quantum Espresso software package [62]. The optimized geometries were introduced into a supercell of $20 \times 20 \times 20 \AA^{3}$. Then, the system of Kohn-Sham equations was solved self-consistently for valence electrons of the clusters to calculate the ground-state eigenvalues using a plane-wave approach [62]. In the calculations, we used a Vanderbilt ultrasoft nonlinear core-corrected pseudopotential [63], which substitutes real valence atomic orbitals in the core region with smooth nodeless pseudo-orbitals [59]. For that, eleven outer-shell electrons $\left(5 d^{10} 6 s^{1}\right)$ of each gold atom were treated as the valence ones. The obtained results were checked by performing a series of calculations with different values of the supercell size and the energy cutoff. The spectra presented in Section III A were obtained with the kinetic energy cutoff of $30 \mathrm{Ry}$ for the wave functions and 180 Ry for the electron densities.

One should note that although the size of the studied systems is not so large (about $1 \mathrm{~nm}$ in diameter), the largest considered cluster, $\mathrm{Au}_{42}$, contains 462 outer-shell electrons, which should be simultaneously accounted for in the DFT/TDDFT calculations. This makes the $a b$ initio-based calculation of the spectra quite demanding
TABLE I. Values of $R_{1}$ and $R_{2}$ which are used to model the electron density distribution in hollow $\mathrm{Au}_{32}$ and $\mathrm{Au}_{42}$ clusters.

\begin{tabular}{lll}
\hline & $R_{1}(\AA)$ & $R_{2}(\AA)$ \\
\hline $\mathrm{Au}_{32}$ & 3.99 & 4.53 \\
$\mathrm{Au}_{42}$ & 4.60 & 5.25 \\
\hline
\end{tabular}

from the computational viewpoint. To analyze the dynamic response of the systems, which are currently used in the experimental studies on sensitization [6-10], one should therefore utilize model approaches. They should also describe adequately the response of smaller systems and must be validated by comparing the results of the $a b$ initio- and model-based calculations.

\section{B. Plasmon resonance approximation}

To describe plasmon excitations arising in gold nanoparticles, we have adopted a simple but physically grounded model which treats the studied highlysymmetric clusters, $\mathrm{Au}_{32}$ and $\mathrm{Au}_{42}$, as a spherical "jellium" shell of a finite width, $\Delta R=R_{2}-R_{1}$. In other words, the electron density in these systems is assumed to be homogeneously distributed over the shell with the thickness $\Delta R$. Such a "jellium"-shell representation has been successfully utilized for the description of plasmon formation in fullerenes $[39,40,44,64-66]$. In this work, we utilize this model for the description of the fullerenelike hollow gold clusters. Values of $\Delta R$ were defined from the analysis of the ground-state geometries of $\mathrm{Au}_{32}$ and $\mathrm{Au}_{42}$. This analysis revealed that the atoms are located on two concentric spheres of the radii $R_{1}$ and $R_{2}$ (see Table I).

Within the PRA, the dynamic polarizability $\alpha(\omega)$ has a resonance behavior in the region of frequencies where collective electron modes in a many-electron system are excited. Thus, the photoionization cross section $\sigma_{\gamma}(\omega)$ of a spherically symmetric system can be written as:

$$
\sigma_{\gamma}(\omega)=\frac{4 \pi \omega}{c} \operatorname{Im} \alpha(\omega) \sim \frac{\omega^{2} \Gamma}{\left(\omega^{2}-\omega_{r}^{2}\right)^{2}+\omega^{2} \Gamma^{2}},
$$

where $\omega$ is the photon energy, $\omega_{r}$ the plasmon resonance frequency, and $\Gamma$ its width. The interaction of a hollow system with the uniform external field, $\mathbf{E}(\omega)$, leads to the variation of the electron density $\delta \rho(\mathbf{r}, \omega)$ occurring on the inner and outer surfaces of the hull. This variation leads to the formation of the surface plasmon, which has two normal modes, the symmetric and antisymmetric ones [64-67]. It has been argued previously [33, 35, 67, 68] that only the surface plasmon can occur in the system interacting with a uniform external electric field, as it happens in the photoionization process. When a system interacts with a non-uniform electric field created, for instance, in collision with a charged particle, the volume plasmon can also occur due to a local compression of the 
electron density in the shell interior [35, 38]. Thus, in the case of irradiation of hollow gold clusters by dipole photons, two surface plasmon modes, characterized by resonance frequencies $\omega_{r}=\omega^{(s)}$ and $\omega_{r}=\omega^{(a)}$ and the widths $\Gamma^{(s)}$ and $\Gamma^{(a)}$, are formed. The frequencies are defined as [64-66]:

$$
\omega^{(s / a)}=\left[\frac{N^{(s / a)}}{2\left(R_{2}^{3}-R_{1}^{3}\right)}(3 \mp p)\right]^{1 / 2},
$$

where the signs '-' and ' + ' correspond to the symmetric $(s)$ and antisymmetric $(a)$ surface mode, respectively, $p=$ $\sqrt{1+8 \xi^{3}}$ with $\xi=R_{1} / R_{2}$ being the ratio of the inner to the outer radius. The values

$$
N^{(s)}=N \frac{p+1}{2 p}, \quad N^{(a)}=N \frac{p-1}{2 p}
$$

are the number of delocalized electrons which are involved in each plasmon mode. They obey the sum rule $N^{(s)}+N^{(a)}=N$ where $N$ stands for a total number of delocalized electrons in the system.

As indicated above, interaction of the system with a non-uniform electric field leads also to the formation of the volume plasmon, which appears due to compression of the electron density inside the volume of the shell and, therefore, does not interfere with either of the surface plasmon modes. With neglect of the dispersion, the volume plasmon frequency $\omega_{p}$, associated with the groundstate electron density $\rho_{0}$ of $N$ electrons, is given by

$$
\omega_{p}=\sqrt{4 \pi \rho_{0}}=\sqrt{3 N /\left(R_{2}^{3}-R_{1}^{3}\right)} .
$$

The formation of the volume plasmon in the electron impact ionization of metal clusters and carbon fullerenes was revealed in Ref. [38, 44, 45]. The model accounting for the contribution of different plasmon modes was successfully utilized to describe the experimentally observed variation of the electron energy loss spectra of $\mathrm{C}_{60}$ in collision with fast electrons $[44,45]$. A detailed explanation of the formation of different plasmon modes can be found in Ref. [33, 35].

\section{RESULTS AND DISCUSSION}

\section{A. Photoabsorption spectra of gold clusters}

The photoabsorption spectra of the $\mathrm{Au}_{18}, \mathrm{Au}_{20}, \mathrm{Au}_{32}$ and $\mathrm{Au}_{42}$ clusters calculated by means of TDDFT for the photon energy up to $60 \mathrm{eV}$ are presented in Figure 2. The spectra, having a similar profile, are characterized by a low-energy peak located below $10 \mathrm{eV}$ and by a broad feature which has the maximum at about $20-25 \mathrm{eV}$. Our analysis has revealed that this feature is the giant resonance formed due to the excitation of electrons in the $5 d$ atomic shell. To prove this, we have integrated the oscillator strength in the photon energy range from
$20.2 \mathrm{eV}$ (ionization threshold of the $5 d$ shell in a single atom of gold) up to $57.2 \mathrm{eV}$, which is the ionization threshold of the $5 p$ shell [69]. The obtained values are $139.8,153.3,240.5$ and 318.7 for $\mathrm{Au}_{18}, \mathrm{Au}_{20}, \mathrm{Au}_{32}$ and $\mathrm{Au}_{42}$, respectively. This indicates that about eight atomic $d$-electrons contribute to the excitation of the $5 d$ shell forming the broad resonance peak in the photoionization spectra. The $5 d$ giant resonance can be fitted with a Fano resonance profile [70],

$$
\sigma_{5 d}(\omega) \equiv \sigma_{5 d \rightarrow \varepsilon p, \varepsilon f}(\omega) \propto \frac{\left(\Gamma_{5 d}+\omega-\omega_{5 d}\right)^{2}}{\left(\Gamma_{5 d} / 2\right)^{2}+\left(\omega-\omega_{5 d}\right)^{2}},
$$

which is frequently utilized in atomic, nuclear and condensed matter physics to describe resonant scattering processes occurring in various systems. Here, $\omega_{5 d}$ stands for the resonance frequency and $\Gamma_{5 d}$ is the width of the peak. To describe this feature, we have utilized the values $\omega_{5 d}=22 \mathrm{eV}$ and $\Gamma_{5 d}=12 \mathrm{eV}$. The fitting for the $\mathrm{Au}_{32}$ cluster is illustrated in Figure 3 by the thick solid purple line.

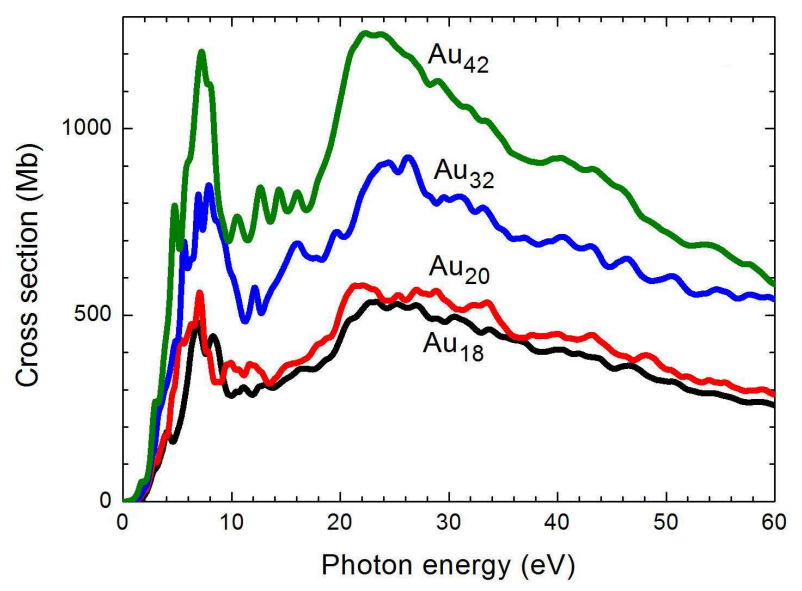

FIG. 2. The photoabsorption cross section of the $\mathrm{Au}_{18}$ (black), $\mathrm{Au}_{20}$ (red), $\mathrm{Au}_{32}$ (blue) and $\mathrm{Au}_{42}$ (green) clusters calculated within the TDDFT framework.

The low-energy peak is related to the surface plasmon, which arises due to collective excitation of delocalized electrons in a whole cluster. The integration of the oscillator strength in this energy region reveals that about $1.0\left(\right.$ in $\mathrm{Au}_{20}$ ) to $1.5\left(\mathrm{in} \mathrm{Au}_{32}\right.$ ) electrons from each atom contribute to the plasmon. Integration of the photoabsorption spectrum of $\mathrm{Au}_{32}$ up to $11.2 \mathrm{eV}$ (energy at which the first dip after the resonance peak is observed) yields the oscillator strength of 1.43 per atom. The collective nature of the low-energy peak is analyzed in more detail in Section III B.

We have fitted the low-energy peak by means of the PRA scheme. In the case of $\mathrm{Au}_{32}$, we have utilized the value $N=46$, assuming that 1.43 electrons from each of 32 atoms are involved in the plasmon excitation. The values of $\Gamma^{(s)}$ and $\Gamma^{(a)}$ were chosen to get the best agreement of the model-based curve with the TDDFT one. 


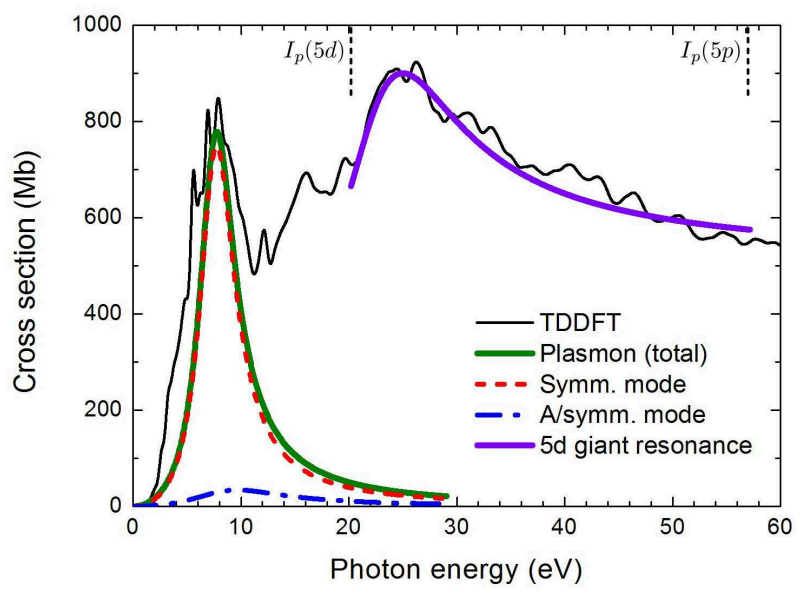

FIG. 3. Photoabsorption cross section of $\mathrm{Au}_{32}$ calculated within the TDDFT method (thin black curve). Thick green curve denotes the contribution of the plasmon excitations calculated within the PRA. Red (dashed) and blue (dash-dotted) curves show the contribution of the symmetric and antisymmetric plasmon modes, respectively. Thick purple line illustrates the fit of the $5 d$ giant atomic resonance by a Fano-type profile. Black vertical marks denote the ionization potentials of the $5 d$ and $5 p$ atomic shells.

In Figure 3, the thick solid (green) curve represents the total plasmon contribution to the cross section. The red and the blue curves illustrate the symmetric and antisymmetric modes, respectively. In this calculation, we have utilized the values $\Gamma^{(s)}=4.0 \mathrm{eV}$ and $\Gamma^{(a)}=10.5 \mathrm{eV}$. The ratio of the widths, $\Gamma^{(s)} / \Gamma^{(a)}=0.38$, is close to the value of 0.34 , which was utilized for the description of the plasmon excitations in fullerenes [39, 45]. Note that the $a b$ initio-based spectrum reveals a more detailed structure which is formed atop the plasmon resonance. This structure represents a series of individual peaks appearing due to single-particle excitations [39] which are neglected in the model.

The oscillator strength, calculated by means of TDDFT in the photon energy range up to the $5 p$ ionization threshold $(\omega=57.2 \mathrm{eV})$, is equal to 338 . This value agrees with the total number of valence electrons in the $\mathrm{Au}_{32}$ cluster, $N=352$, with the relative discrepancy of about 5 percent. As mentioned above, we assume that about 1.5 and 8 electrons from each atom contribute to the surface plasmon and the $5 d$ giant resonance, respectively. Thus, we have accounted for the contribution of 9.5 from 11 valence electrons from each atom. The contribution of the rest results in a series of individual peaks, positioned in the photon energy range from 11.2 (the first dip after the low-energy peak) to $20.2 \mathrm{eV}$ (the $5 d$ ionization threshold), that are not accounted for in our model analysis. Integration of this part of the spectrum yields the oscillator strength of 51.5, i.e. 1.6 electrons from each atom contribute to the excitations in this energy region. The individual peaks appear due to single-particle excitations from the $s-d$ band formed due to hybridiza- tion of the $6 s$ and $5 d$ atomic shells. On the basis of the performed analysis, the total photoabsorption spectra of gold clusters in the energy region up to $60 \mathrm{eV}$ can be approximated by the sum of the plasmon contribution and that of the $5 d$ electron excitations in individual atoms, $\sigma_{\gamma} \approx \sigma_{\mathrm{pl}}+\sigma_{5 \mathrm{~d}}$.

In order to stress the different nature of the low- and high-energy features in the photoabsorption spectra of the clusters, we performed an additional comparison with the spectrum of a smaller molecular system, a gold dimer. Figure 4 demonstrates the spectra of $\mathrm{Au}_{32}$ and $\mathrm{Au}_{2}$ normalized to the number of atoms in each system. The spectra have a similar profile in the energy region above $15 \mathrm{eV}$. This indicates the same origin of excitations in the dimer and in larger atomic clusters, that is related to the excitation of electrons in the $5 d$ shell. The ripple, which is seen at high photon energies, has an artificial origin and arises due to the method of calculation of the dipole susceptibility, which is obtained at complex frequencies with an imaginary part of $0.5 \mathrm{eV}$. The normalized spectra are compared also to the $\mathrm{x}$-ray absorption data for atomic gold taken from the Henke tables [69]. We state a consistency of the TDDFT-based spectra and well-established set of data.

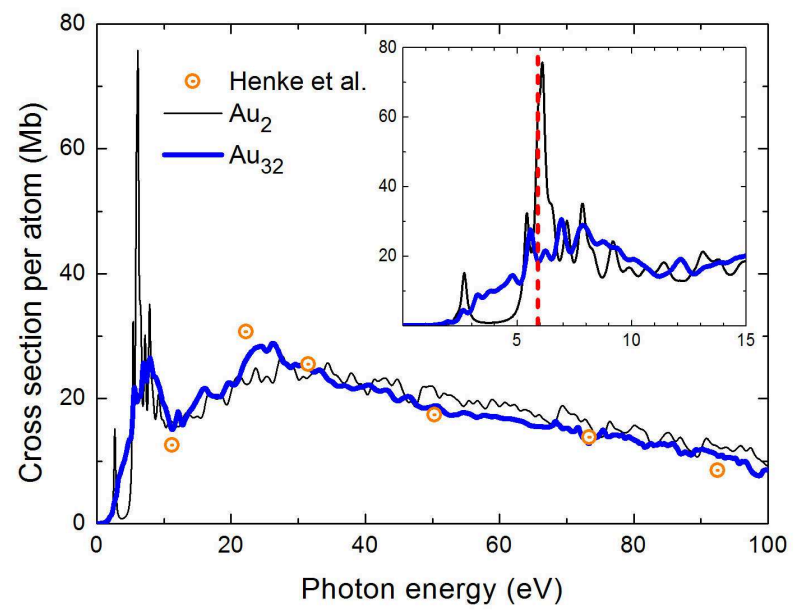

FIG. 4. The normalized photoabsorption cross section of $\mathrm{Au}_{32}$ (thick blue curve) and of the $\mathrm{Au}_{2}$ molecule (thin black curve) calculated within the TDDFT framework. Symbols represent the x-ray absorption data for atomic gold compiled by Henke et al. [69]. The inset shows the low-energy part of the spectra. Red vertical line illustrates the ionization threshold of a single gold atom as obtained from the DFT calculations.

On the contrary, the low-energy part of the spectrum is quite different in the two considered cases. The cross section of the dimer is represented by several well-resolved narrow peaks due to particular molecular transitions, as opposed to a broader feature appeared in the spectrum of $\mathrm{Au}_{32}$. The inset of Figure 4 shows that the low-energy part of the spectrum of $\mathrm{Au}_{2}$ is described by the two peaks, positioned at 2.7 and $5.4 \mathrm{eV}$, followed by the prominent peak at $6.1 \mathrm{eV}$. The latter peak corresponds to ionization 
of the molecule (the ionization threshold of $\mathrm{Au}_{2}$ is marked by the dashed vertical line). Therefore, one can observe a clear difference in the structure of the two spectra below $10 \mathrm{eV}$, that indicates two different mechanisms of electronic excitations arising in this energy region, namely well-resolved molecular transitions in the case of $\mathrm{Au}_{2}$ and the collective excitation of delocalized electrons in the $\mathrm{Au}_{32}$ cluster. In the following section, we analyze the difference in the structure of the valence band of $\mathrm{Au}_{32}$ and $\mathrm{Au}_{2}$ in greater detail.

\section{B. Plasmon nature of the low-energy peak}

Here, we provide an explanation of why we attribute the low-energy peak in the photoabsorption spectra of gold clusters to a plasmon-type excitation.

The term "plasmon" is generally used to describe a collective excitation of delocalized electrons of a system to an external electromagnetic field. Quite commonly, it is understood in terms formulated, for example, by Fano. In his review [71] on collective phenomena in nanoscale systems and in condensed matter, it is stated that "...common to these phenomena [plasmons, superconductivity, etc., i.e. those phenomena which are based on the motion of (quasi)independent particles] is the role of a dense spectrum of states viewed initially as independent. The seemingly weak interaction among these states often condenses into a single eigenvalue separated from the rest of the spectrum by an energy gap". Thus, if a system has a dense packing of states with a small state separation, the excitation of these states may be considered as a collective, plasmon-type one.

In a number of papers [72-75], the term "surface plasmon resonance" (SPR) in relation to gold nanoparticles describes a peak in the visible part of the absoprtion spectra (at about $2.3 \mathrm{eV}$ ). It was stated that this feature is caused by the collective excitation of $6 s$ electrons in the gold atoms [72-75]. The corresponding electronic levels are located in the vicinity of the Fermi surface, so that these electrons delocalize over the whole nanoparticle. It was also stated $[72,73]$ that such a SPR (also referred to as the "localized SPR", LSPR) is a characteristic feature of relatively large systems, while the threshold nanoparticle size for emergence of such plasmonic absorption is about $1.5-2 \mathrm{~nm}$ (this value corresponds to the number of atoms in the system of about $150-200)$. Smaller gold nanoparticles (less than $1 \mathrm{~nm}$ in diameter) should have discrete energy levels and, thus, molecular-type transitions between the occupied and unoccupied states [74].

On the other hand, it is also well acknowledged that the occupied $6 s$ states in the gold atoms are strongly hybridized with the $5 d$ orbitals. A general remark on this issue is made in Ref. [75] that "...almost always a rather strong mixing is observed, this finding reveals the "collective" nature of the electron excitation".

In Figure 5, we compare the dipole oscillator strength distribution of the $\mathrm{Au}_{32}$ cluster and of the $\mathrm{Au}_{2}$ molecule.
The low-energy peak in the spectrum of $\mathrm{Au}_{32}$ corresponds to the ionization of the valence band whose structure is shown in the inset. The valence orbitals in $\mathrm{Au}_{32}$ span over the energy range of about $5.8 \mathrm{eV}$, so that the HOMO and the innermost valence state have the ionization potentials of 5.77 and $11.57 \mathrm{eV}$, respectively. The $6 s$ and $5 d$ orbitals are hybridized and degenerated according to the cluster symmetry. Since $\mathrm{Au}_{32}$ is a highly-symmetric structure possessing icosahedral symmetry [51, 52], its molecular orbitals are singly, triply, fourfold and fivefold degenerated in accordance with the irreducible representation of the $I_{h}$ point group [76]. In $\mathrm{Au}_{32}$, there are 176 valence orbitals which are reduced to 46 because of symmetry of the cluster. Analysis of the valence state separation indicates that this value varies from 0.01 to $0.64 \mathrm{eV}$, resulting in the average value of $0.13 \mathrm{eV}$. This value corresponds to those calculated [77] for a number of silver clusters $\operatorname{Ag}_{n}(n=20 \ldots 120)$. The emergence of a plasmon peak was observed in these systems by means of the TDDFT approach.

Based on the analysis of the valence band structure and on the explanation given by Fano [71], we thus associate the low-energy peak in the photoabsorption spectra of the gold clusters with a collective, plasmon-type excitation. However, we note that this excitation involves not only the $6 s$ electrons (as it happens in the case of the LSPR located at about $2.3 \mathrm{eV}$ [72-75]) but also some fraction of the $5 d$ electrons because of the strong overlap between the $s$ and $d$ states.

To the further support of this statement, we have analyzed this collective excitation in terms of the classical Mie theory. The $\mathrm{Au}_{32}$ cluster has a hollow, fullerene-like structure so that the electron density is assumed to be homogeneously distributed in between the two spheres on which the atoms are positioned. Calculating the frequency of a surface plasmon excited in a hull-like system with the help of Eq. (9), one derives the value of $6.3 \mathrm{eV}$ that matches well the position of the dominant feature in the TDDFT-based spectrum of $\mathrm{Au}_{32}$. Thus, we can state that the low-energy peak arises due to the collective, plasmon-type excitation of electrons delocalized over the whole cluster. In this estimate, we assumed that 46 electrons (1.43 electrons from each atom) delocalize and participate in the collective excitation. This value is in agreement with the results of Ref. [78], where the dipole polarizability of a series of three-dimensional gold clusters as a function of their size was calculated. It was found that the calculated polarizabilities suggest a delocalized character of some fraction of $d$ electrons, so that 1.56 delocalized valence electrons contribute to the linear response to an external field.

\section{Electron production via the plasmon excitation mechanism}

Having justified the parameters of the model that describe the dipole plasmon excitation in gold nanoparti- 

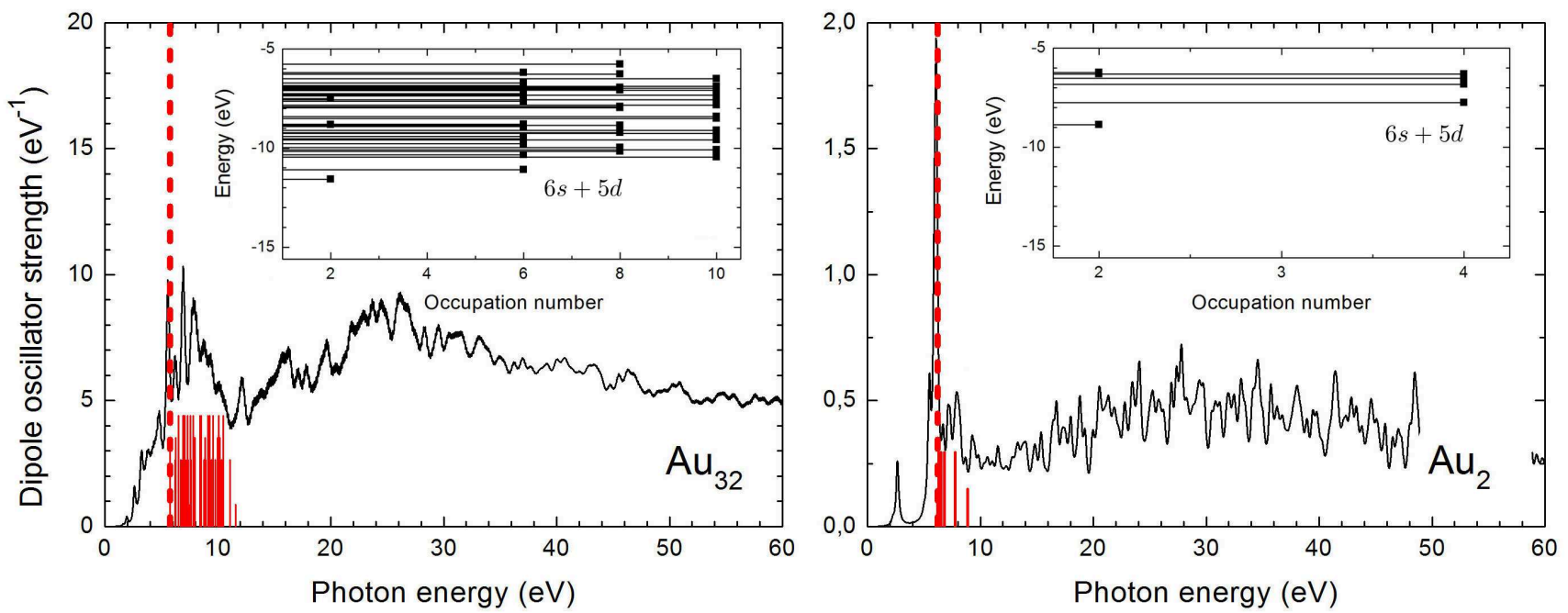

FIG. 5. Dipole oscillator strength of the $\mathrm{Au}_{32}$ cluster (left) and of $\mathrm{Au}_{2}$ dimer (right) calculated within the TDDFT framework. Vertical red lines mark ionization thresholds of each of the $6 s+5 d$ valence states. Thick dashed lines denote the ionization threshold of the HOMO state in each system. Inset: Single-electron energy levels of the valence band as calculated at CEP$121 \mathrm{G}(\mathrm{d}) / \mathrm{PBEPBE}$ level of theory.

cles, we extend the model approach to study the electron production due to the plasmon excitation mechanism. Within the PRA, the double differential inelastic scattering cross section of a fast projectile in collision with a hull-like system can be defined as a sum of three terms $[35,44]$ :

$$
\frac{\mathrm{d}^{2} \sigma_{\mathrm{pl}}}{\mathrm{d} \varepsilon_{2} \mathrm{~d} \Omega_{\mathbf{p}_{2}}}=\frac{\mathrm{d}^{2} \sigma^{(s)}}{\mathrm{d} \varepsilon_{2} \mathrm{~d} \Omega_{\mathbf{p}_{2}}}+\frac{\mathrm{d}^{2} \sigma^{(a)}}{\mathrm{d} \varepsilon_{2} \mathrm{~d} \Omega_{\mathbf{p}_{2}}}+\frac{\mathrm{d}^{2} \sigma^{(v)}}{\mathrm{d} \varepsilon_{2} \mathrm{~d} \Omega_{\mathbf{p}_{2}}}
$$

which describe the partial contribution of the surface (the two modes, $s$ and $a$ ) and the volume $(v)$ plasmons. Here $\varepsilon_{2}$ is the kinetic energy of the scattered projectile, $\mathbf{p}_{2}$ its momentum, and $\Omega_{\mathbf{p}_{2}}$ its solid angle. The cross section $\mathrm{d}^{2} \sigma_{\mathrm{pl}} / \mathrm{d} \varepsilon_{2} \mathrm{~d} \Omega_{\mathbf{p}_{2}}$ can be written in terms of the energy loss $\Delta \varepsilon=\varepsilon_{1}-\varepsilon_{2}$ of the incident projectile of energy $\varepsilon_{1}$. Integration of $\mathrm{d}^{2} \sigma_{\mathrm{pl}} / \mathrm{d} \Delta \varepsilon \mathrm{d} \Omega_{\mathbf{p}_{2}}$ over the solid angle leads to the single differential cross section:

$$
\frac{\mathrm{d} \sigma_{\mathrm{pl}}}{\mathrm{d} \Delta \varepsilon}=\int \mathrm{d} \Omega_{\mathbf{p}_{2}} \frac{\mathrm{d}^{2} \sigma_{\mathrm{pl}}}{\mathrm{d} \Delta \varepsilon \mathrm{d} \Omega_{\mathbf{p}_{2}}}=\frac{2 \pi}{p_{1} p_{2}} \int_{q_{\min }}^{q_{\max }} q \mathrm{~d} q \frac{\mathrm{d}^{2} \sigma_{\mathrm{pl}}}{\mathrm{d} \Delta \varepsilon \mathrm{d} \Omega_{\mathbf{p}_{2}}},
$$

where $\mathbf{p}_{1}$ is the initial momentum of the projectile and $\mathbf{q}=\mathbf{p}_{1}-\mathbf{p}_{2}$ the transferred momentum. Explicit expressions for the contributions of the surface and the volume plasmons, entering Eq. (13), obtained within the planewave Born approximation, are presented in Ref. [35]. The Born approximation is applicable since the considered collision velocities $(v=2-20$ a.u. $)$ are significantly larger than the characteristic velocities of delocalized electrons in the gold nanoparticles $(v \approx 0.5$ a.u. $)$.

The surface and the volume plasmon terms appearing on the right-hand side of Eq. (13) are constructed as a sum over different multipole contributions corresponding to different values of the angular momentum $l$ :

$$
\begin{aligned}
\frac{\mathrm{d}^{2} \sigma^{(i)}}{\mathrm{d} \varepsilon_{2} \mathrm{~d} \Omega_{\mathbf{p}_{2}}} \propto \sum_{l} \frac{\omega_{l}^{(i) 2} \Gamma_{l}^{(i)}}{\left(\omega^{2}-\omega_{l}^{(i) 2}\right)^{2}+\omega^{2} \Gamma_{l}^{(i) 2}} \\
\frac{\mathrm{d}^{2} \sigma^{(v)}}{\mathrm{d} \varepsilon_{2} \mathrm{~d} \Omega_{\mathbf{p}_{2}}} \propto \sum_{l} \frac{\omega_{p}^{2} \Gamma_{l}^{(v)}}{\left(\omega^{2}-\omega_{p}^{2}\right)^{2}+\omega^{2} \Gamma_{l}^{(v) 2}},
\end{aligned}
$$

where $i=s, a$ denotes the two modes of the surface plasmon. The frequencies of the symmetric and antisymmetric modes of the multipolarity $l$ are given by $[35,65]$ :

$$
\omega_{l}^{(s / a)}=\left(1 \mp \frac{1}{2 l+1} \sqrt{1+4 l(l+1) \xi^{2 l+1}}\right)^{1 / 2} \frac{\omega_{p}}{\sqrt{2}}
$$

where ' - ' and ' + ' stand for symmetric $(s)$ and antisymmetric $(a)$ modes, respectively. In the dipole case $(l=1)$, this expression reduces to Eq. (9). The volume plasmon frequency, $\omega_{p}$, is independent on $l$ as it follows from Eq. (11).

Figure 6 shows the cross section $\mathrm{d} \sigma_{\mathrm{pl}} / \mathrm{d} \Delta \varepsilon$ calculated for the $\mathrm{Au}_{32}$ cluster irradiated by fast protons of different incident energies as indicated. The figure demonstrates that the amplitude and the shape of the plasmon resonance depend strongly on the kinetic energy of the proton. It was shown previously [41] that the relative contribution of the quadrupole $(l=2)$ and higher multipole terms to the cross section decreases significantly with an increase of the collision velocity. At high velocities, the dipole contribution dominates over the higher multipole contributions, since the dipole potential decreases slower at large distances than the higher multipole potentials.

The presented spectra comprise contributions of both the surface and the volume plasmon excitations, and different multipole terms contribute to each of them. Calculating the cross sections presented in Figure 6, we 


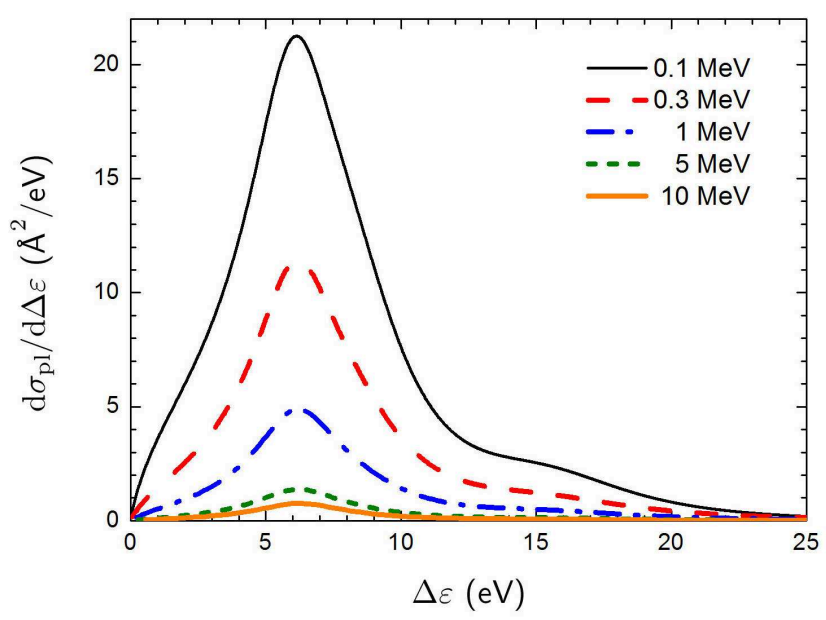

FIG. 6. Contribution of the plasmon excitations to the single differential cross section, $\mathrm{d} \sigma_{\mathrm{pl}} / \mathrm{d} \Delta \varepsilon$, of the $\mathrm{Au}_{32}$ cluster irradiated by fast protons of different incident energies as a function of the energy loss.

accounted for the contribution of the dipole $(l=1)$, quadrupole $(l=2)$ and octupole $(l=3)$ terms. The excitations with large angular momenta have a single-particle rather than a collective nature [41]. With increasing $l$, the wavelength of a plasmon excitation, $\lambda_{\mathrm{pl}}=2 \pi R / l$, becomes smaller than the characteristic wavelength of the delocalized electrons in the system, $\lambda_{e}=2 \pi / \sqrt{2 \epsilon}$. Here $\epsilon \sim I_{p}$ is the characteristic electron excitation energy in the cluster, and $I_{p}$ is the ionization threshold of the system. The DFT calculations of the electronic structure of $\mathrm{Au}_{32}$ derived the value of $I_{p}=5.77 \mathrm{eV}$, while the calculated HOMO-LUMO gap is $1.54 \mathrm{eV}$. Thus, one estimates a characteristic excitation energy of delocalized electrons in $\mathrm{Au}_{32}$ to be of the order of several electronvolts, that results in the account of the three multipole plasmon terms.

Following the methodology utilized in Ref. [45], we assume that the ratio $\gamma_{l}=\Gamma_{l} / \omega_{l}$ of the width of the plasmon resonance to its frequency equals to $\gamma_{l}^{(s)}=0.6$ for all multipole terms of the symmetric mode, and to $\gamma_{l}^{(a)}=1.0$ for the antisymmetric mode. In Ref. $[39,45]$ these values were successfully utilized to describe the main features of the photon and electron impact ionization cross sections of the $\mathrm{C}_{60}$ fullerene whose topology is similar to the $\mathrm{Au}_{32}$ cluster. For the volume plasmon we consider the ratio $\gamma_{l}^{(v)}=\Gamma_{l}^{(v)} / \omega_{p}=1.0$.

Figure 7 illustrates the contribution of different plasmon modes to the spectrum of $\mathrm{Au}_{32}$ irradiated by a 1 $\mathrm{MeV}$ proton and also partial contributions of different multipole modes. The main contribution to the cross section comes from the symmetric mode of the surface plasmon, which, in turn, is dominated by the dipole excitation. The figure shows that the relative contribution of the surface plasmon exceeds that of the volume plasmon by more than an order of magnitude. Thus,
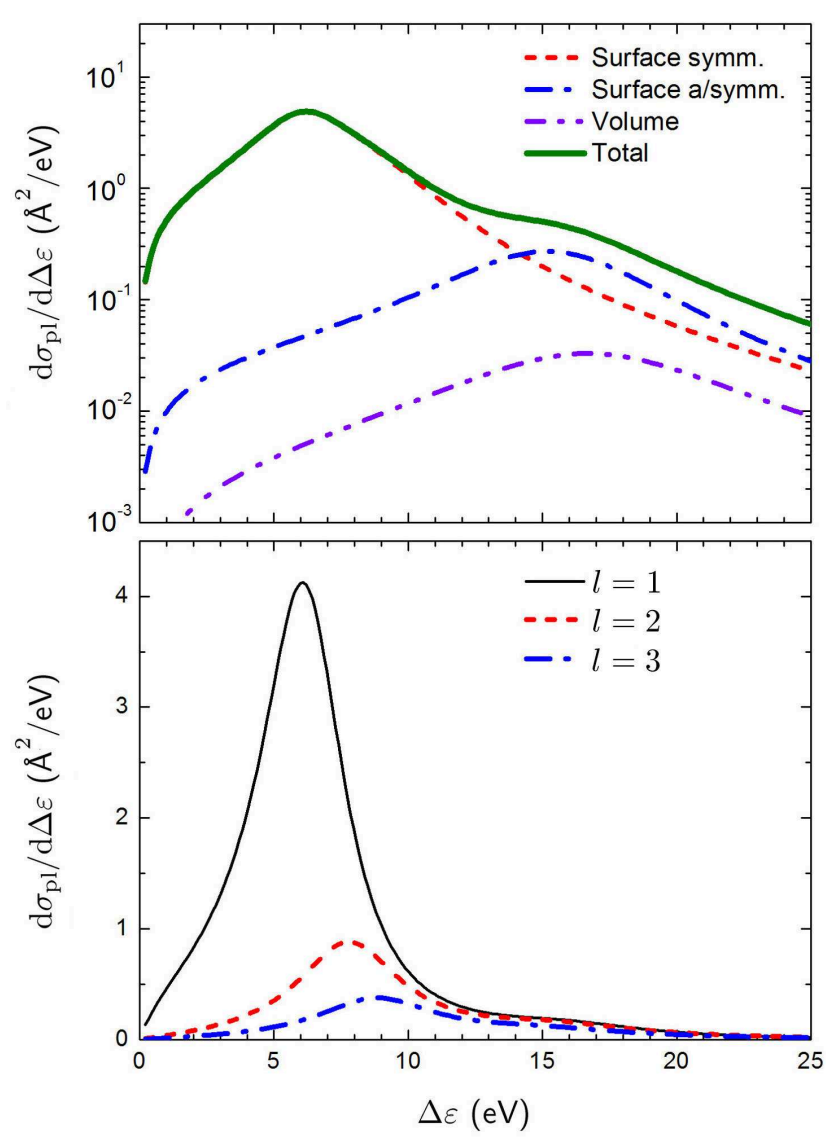

FIG. 7. Single differential cross section $\mathrm{d} \sigma_{\mathrm{pl}} / \mathrm{d} \Delta \varepsilon$ of the $\mathrm{Au}_{32}$ cluster irradiated by a $1 \mathrm{MeV}$ proton as a function of the energy loss. Upper panel illustrates the contribution of different plasmon excitations to the resulting spectrum. Lower panel shows the contribution of different multipole terms.

the leading mechanism of electron production by gold nanoparticles is related to the surface plasmon. This result contradicts with the recent Monte Carlo simulations [28], which claimed that the plasmon excitations do not play an important role in the process of electron emission from metallic nanoparticles. Let us stress that only the volume plasmon excitation was accounted for in those simulations. Below we demonstrate that the emission of the low-energy electrons from the gold nanoparticles is indeed a prominent effect, which should be accounted for when estimating the secondary electron yield in a biological medium with embedded nanoparticles.

Note that the maximum of the resonance peak is located at $6.3 \mathrm{eV}$, that is slightly above the ionization threshold of $\mathrm{Au}_{32}, I_{p}=5.77 \mathrm{eV}$. The plasmons located above the ionization threshold can decay via the ionization process [38]. On the contrary, the decay of a collective excitation located below the ionization threshold results in single-electron excitations, which can also be coupled with the ionic motion by the electron-phonon coupling [79]. Therefore, decay of the surface plasmon in $\mathrm{Au}_{32}$ results in the electron emission from the system 
which can be accompanied by vibrations of the ionic core.

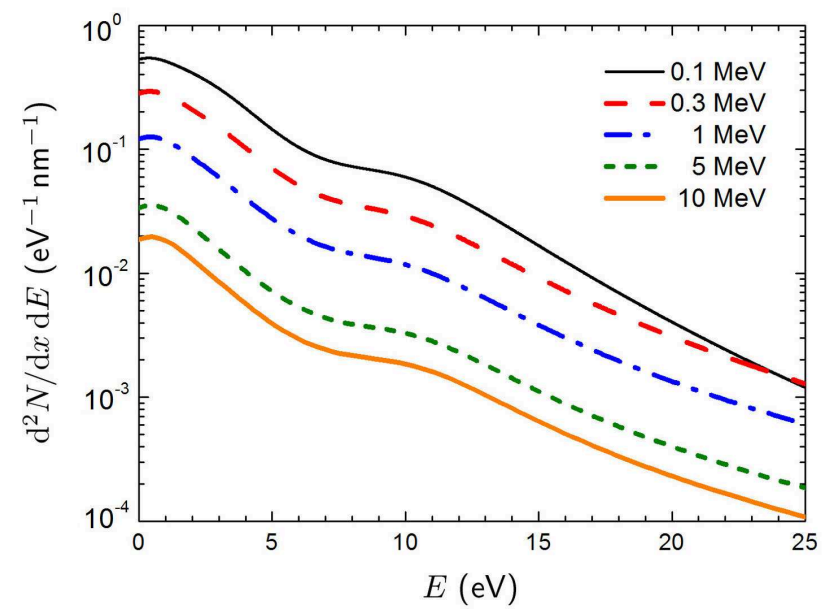

FIG. 8. Number of electrons per unit length per unit energy emitted via the plasmon excitation mechanism from the $\mathrm{Au}_{32}$ cluster irradiated by a fast proton. Different curves illustrate different values of the proton's kinetic energy.

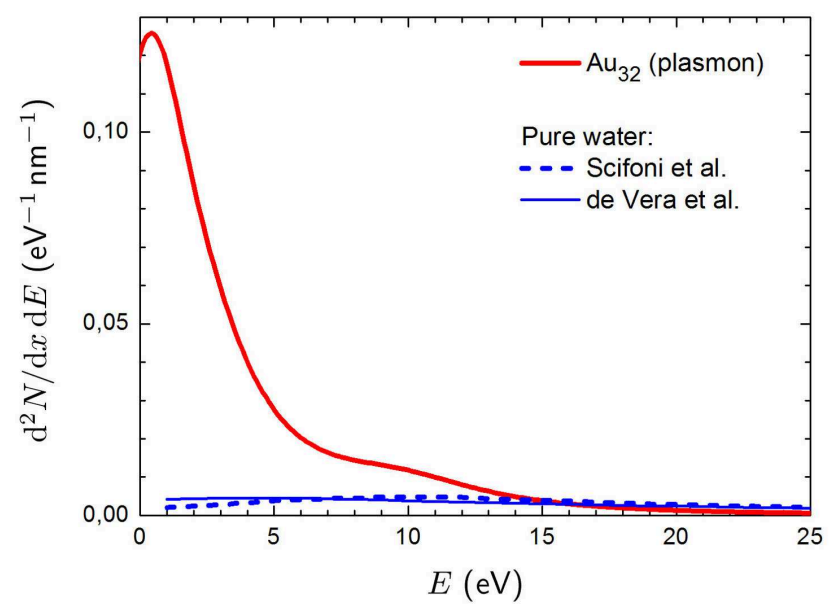

FIG. 9. Number of electrons per unit length per unit energy emitted via the plasmon excitation mechanism from the $\mathrm{Au}_{32}$ cluster irradiated by a $1 \mathrm{MeV}$ proton (red curve). Blue curves represent the number of electron generated from an equivalent volume of pure water. Solid and dashed blue curves represent the results obtained within the dielectric formalism by Scifoni et al. [80] and de Vera et al. [81], respectively.

To quantify the production of electrons via the plasmon excitation mechanism, we redefine the cross section $\mathrm{d} \sigma_{\mathrm{pl}} / \mathrm{d} \Delta \varepsilon$ as a function of the kinetic energy $E$ of emitted electrons. This quantity is related to the energy loss via $E=\Delta \varepsilon-I_{p}$, where $I_{p}$ is the ionization threshold of the system. The cross section $\mathrm{d} \sigma_{\mathrm{pl}} / \mathrm{d} E$ can be related to the probability to produce $N$ electrons with kinetic energy $E$, in the interval $d E$, emitted from a segment $\mathrm{d} x$, via
[11]:

$$
\frac{\mathrm{d}^{2} N(E)}{\mathrm{d} x \mathrm{~d} E}=\frac{1}{V} \frac{\mathrm{d} \sigma_{\mathrm{pl}}}{\mathrm{d} E}
$$

where $V$ is the volume occupied by the nanoparticle. Assuming that the linear size of the $\mathrm{Au}_{32}$ cluster is $d \approx 0.9 \mathrm{~nm}$, we have calculated the number of electrons per unit length per unit energy emitted from this system due to proton irradiation. Figure 8 illustrates the dependence of this quantity on kinetic energy of emitted electrons for different incident energies of the proton.

In Figure 9, we compare the electron production by $\mathrm{Au}_{32}$ (red curve) and by an equivalent volume of pure water medium (blue curves) irradiated by a $1 \mathrm{MeV}$ proton. Solid and dashed blue curves represent the results obtained recently within the dielectric formalism [80, 81]. This approach is based on the experimental measurements of the energy-loss function of the target medium, $\operatorname{Im}[-1 / \epsilon(\omega, q)]$, where $\epsilon(\omega, q)$ is the complex dielectric function, with $\omega$ and $q$ being the energy and the momentum transferred to the electronic excitation, respectively. Comparative analysis of the spectra reveals that the number of the low-energy electrons (with the kinetic energy of about a few $\mathrm{eV}$ ) produced by the gold nanoparticle is about one order of magnitude higher than by liquid water.

In the above-presented analysis, we have considered the hollow cluster of diameter $d \approx 0.9 \mathrm{~nm}$. An additional estimate was done also for a solid nanoparticle of a similar size. As a case study, we considered a $1 \mathrm{~nm}$ nanoparticle "cut" from an ideal gold crystal having the face-centered cubic (fcc) lattice with the parameter $a=4.08 \AA$. The crystalline structure was constructed using the "Crystal generator" tool [82] of the MBN Explorer [83] software. As a result, we found that the small solid gold nanoparticle is composed of 31 atoms. Thus, its atomic density is close to that of the above-considered $\mathrm{Au}_{32}$ cluster.

The dynamic response of a solid nanoparticle can also be modeled by means of the PRA formalism assuming that the system is treated not as a "jellium" hull but as a full sphere, where the electron density is uniformly distributed inside the sphere of a radius $R[37,38]$. In this case, the electron density variation on the surface and in the volume of the nanoparticle leads to the formation of the surface (symmetric mode) and the volume plasmon, respectively, while the antisymmetric surface plasmon mode does not contribute to the cross section (a detailed explanation of this phenomenon can be found in Ref. [35]). As shown in Figure 7 in the case of the hollow system, the contribution of the antisymmetric mode in the $1-10 \mathrm{eV}$ range is an order of magnitude smaller than that of the symmetric mode. Thus, the absence of the antisymmetric mode in the excitation spectrum of the solid nanoparticle should not lead to quantitatively different results from those presented in Figure 7. Therefore, all the above-given estimates on the electron production yield by the hollow nanoparticle of about $1 \mathrm{~nm}$ diameter should also be valid for the case of the solid fcc structure. 


\section{Contribution of individual atomic excitations to electron production}

As illustrated above (see Figure 3), there is also a prominent contribution of the atomic $5 d$ electrons to the ionization cross section. The $d$ electrons in the atoms of gold play a dominant role at the excitation energies from approximately 20 to $60 \mathrm{eV}$. For distant collisions, i.e. when the impact parameter exceeds the radius $R_{\text {at }}$ of the atomic subshell, the ionization spectra are dominated by the dipole term [84]. On this basis, we have compared the cross sections of photoionization, $\sigma_{\gamma}$, and the dipole term of atomic inelastic scattering, $\mathrm{d} \sigma_{5 \mathrm{~d}} / \mathrm{d} \Delta \varepsilon$, calculated in the Born approximation. As a result, one derives the following expression:

$$
\frac{\mathrm{d} \sigma_{5 d}}{\mathrm{~d} \Delta \varepsilon}=\frac{2 c}{\pi \omega v_{1}^{2}} \sigma_{\gamma} \ln \left(\frac{v_{1}}{\omega R_{5 d}}\right),
$$

where $\sigma_{\gamma} \equiv \sigma_{5 d}(\omega)$ is the $5 d$ photoionization cross section estimated by a Fano-type profile, Eq. (12), $\omega=\varepsilon_{1}-\varepsilon_{2}$ the energy transfer, $v_{1}$ the projectile velocity, and $R_{5 d}$ a characteristic radius of the $5 d$ electron shell. Equation (18), obtained within the so-called "logarithmic approximation", assumes that the main contribution to the cross section $\mathrm{d} \sigma_{5 \mathrm{~d}} / \mathrm{d} \Delta \varepsilon$ comes from the region of large distances, $R_{5 d}<r<v_{1} / \omega$. This relation has the logarithmic accuracy which implies that the logarithmic term dominates the cross section while all non-logarithmic terms are neglected [48]. Making an estimate for the gold atoms, we assumed $\omega \approx 1$ a.u. which corresponds to the maximum of the $5 d$ giant resonance in gold [69], $v_{1} \approx 6.3$ a.u. for a $1 \mathrm{MeV}$ proton, and the electron shell radius $R_{5 d}(\mathrm{Au}) \approx 2$ a.u. Note that the interaction of the incident projectile with the nanoparticle leads to the formation of the $5 d$ giant resonance not in all atoms of the system but only in those located within the impact parameter interval from $r_{\min } \simeq R_{5 d}$ to $r_{\max } \simeq v_{1} / \omega$. This estimate indicates that the $5 d$ giant resonance is formed in about one third of atoms of the nanoparticle.

The number of electrons per unit length per unit energy produced via the excitation of $5 d$ electrons in individual gold atoms, is defined as:

$$
\frac{\mathrm{d}^{2} N(E)}{\mathrm{d} x \mathrm{~d} E}=A n \frac{\mathrm{d} \sigma_{5 d}}{\mathrm{~d} E},
$$

where $n$ is the atomic density of the target, and $A$ the ratio of the number of atoms possessing the $5 d$ resonance to the total number of atoms in the nanoparticle.

To estimate the total number of electrons produced due to the collective excitations in the gold nanoparticle, we have accounted for the contribution of the plasmon excitations and excitations of $5 d$ electrons in individual atoms. Figure 10 shows the relative enhancement of the electron yield from the $\mathrm{Au}_{32}$ cluster as compared to pure water. The data for the gold nanoparticle are normalized to the spectrum for liquid water [81]. The solid line shows the contribution of the plasmon excitations to the

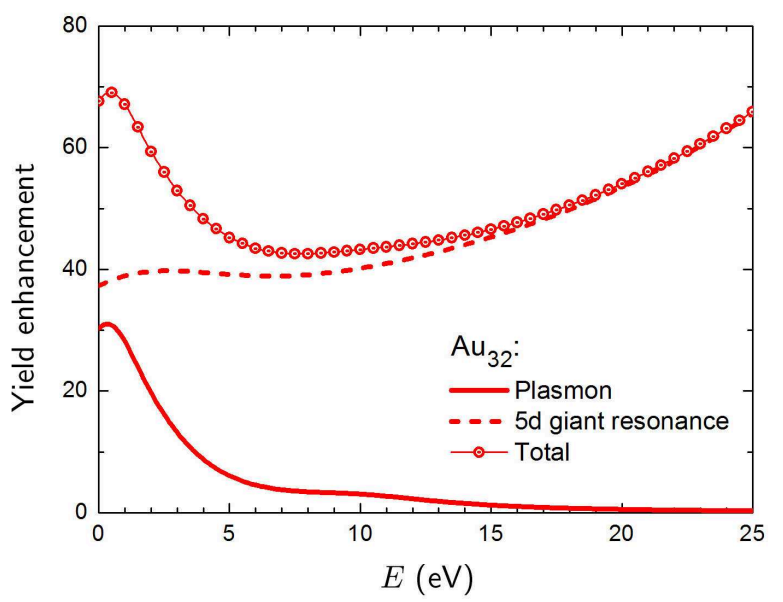

FIG. 10. Yield enhancement from the $\mathrm{Au}_{32}$ cluster as compared to an equivalent volume of pure water [81]. The solid and dashed lines show the contribution of the plasmons and the atomic $5 d$ excitations, respectively. Symbols illustrate the resulting enhancement.

electron yield, while the dashed line presents the contribution from the atomic $5 d$ giant resonance, estimated using Eq. (18) and (19). Making this estimate, we have assumed that the ionization cross sections of individual atoms are dominated by the dipole excitation. Contribution of quadrupole and higher multipole terms will lead to an increase in the number of emitted electrons but their relative contribution will be not as large as that from the dipole excitation. Accounting for the plasmon contribution leads to a significant additional increase in the number of $1-5 \mathrm{eV}$ emitted electrons as compared to the pure water. Due to the collective electron excitations arising in the $\sim 1 \mathrm{~nm}$ gold nanoparticle, it can thus produce up to 70 times larger number of the low-energy electrons comparing to the equivalent volume of pure water medium. The enhancement of the secondary electron yield may increase the probability of the tumor cell destruction due to the double- or multiple strand break of the DNA [11]. The results of the performed analysis indicate that the decay of the collective electron excitations in gold nanoparticles is an important mechanism of the yield enhancement.

\section{E. Different kinematic conditions}

In this section, we analyze how the contribution of the plasmon and the $5 d$ excitation mechanisms evolve at different kinematic conditions, namely for different projectile velocities and for the nanoparticles of different size.

In the case of the $\mathrm{Au}_{32}$ cluster irradiated by a $1 \mathrm{MeV}$ proton, the number of electrons produced via the excitations in individual atoms is generally higher than that produced via the plasmon excitation mechanism (see Figure 10). At certain kinematic conditions, the plasmon contribution to the low-energy (of about $1-10 \mathrm{eV}$ ) elec- 
tron yield from the gold nanoparticle can exceed significantly that due to the atomic giant resonance. An illustration of this effect is given in Figure 11, where we compare the yield of electron production due to irradiation by 1 and $0.1 \mathrm{MeV}$ protons. We also consider the incident energy of $0.3 \mathrm{MeV}$ which is approximately equal to that of an ion in the vicinity of the Bragg peak [85, 86]. The electron yield due to the plasmon excitations grows with decreasing the projectile's energy (see also Figure 8). On the contrary, the yield due to the atomic excitations exhibits a different behavior. The number of electrons emitted from the $\mathrm{Au}_{32}$ cluster via the decay of the atomic $5 d$ excitation by a $0.3 \mathrm{MeV}$ proton is larger than in the case of a $1 \mathrm{MeV}$ projectile. However, a decrease of incident energy down to $0.1 \mathrm{MeV}$ leads to an abrupt decrease of the number of produced electrons. As follows from Eq. (18), at $\varepsilon_{1}=0.1 \mathrm{MeV}\left(v_{1}=2.00073\right.$ a.u. $)$, the dipole term of the $5 d$ inelastic scattering cross section is strongly suppressed, as the $\ln \left(v_{1} / \omega R_{5 d}\right)$ term approaches zero. In this case, the yield of electrons with kinetic energy below $5 \mathrm{eV}$ due to the plasmon excitation exceeds that due to the $5 d$ atomic excitation by the factor of about $10^{3}$.

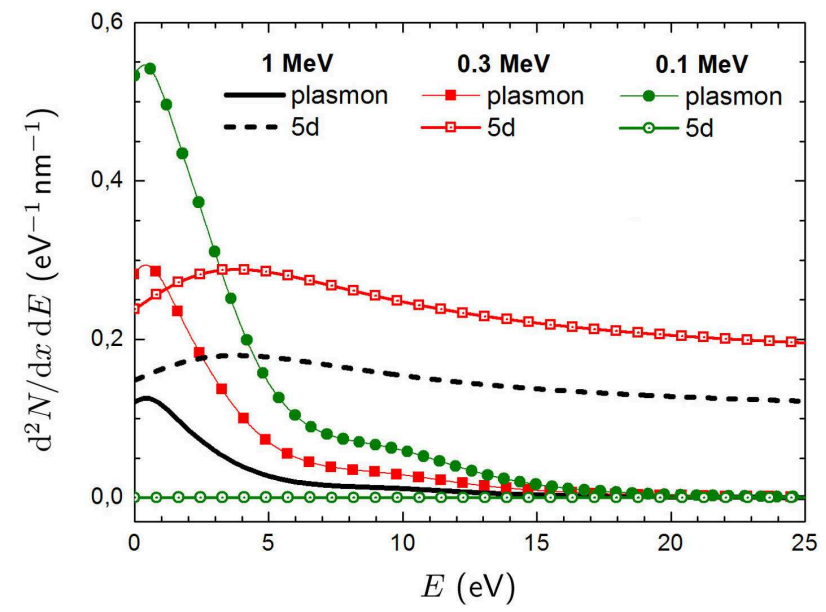

FIG. 11. Number of electrons per unit length per unit energy produced via the plasmon and the $5 d$ excitation mechanisms in the $\mathrm{Au}_{32}$ cluster irradiated by a proton of different kinetic energies. Solid line and filled symbols illustrate the plasmon contribution to the electron production yield. Dashed line and open symbols show the contribution of the $5 d$ giant resonance.

Finally, let us analyze the role of the nanoparticle size on the intensity of electron production. In Figure 12, we consider the electron yield from the solid gold nanoparticles of different size irradiated by the $0.1,0.3,1$ and $10 \mathrm{MeV}$ protons. We focus on the systems of about $1-10$ $\mathrm{nm}$ in diameter. Metal nanoparticles of this size range were studied recently in relation to the radiotherapies with charged ions [6, 10]. For the sake of clarity, we have calculated the number of electrons per unit energy, $\mathrm{d} N / \mathrm{d} E$. At certain kinematic conditions, the contribution of the plasmon excitations saturates, so that larger nanoparticles emit a smaller number of electrons via the plasmon damping mechanism.

It was shown previously [41] that the dipole mode of the plasmon excitations arising in a nanoparticle gives the dominating contribution to the ionization cross section when the characteristic collision distance exceeds significantly the nanoparticle size, $v_{1} / \omega \gg D / 2$ where $D$ is the nanoparticle diameter. At large collision distances, the dipole contribution dominates over the higher multipole contributions. Terms with higher $l$ become significant only in the case when the collision distances become comparable with the cluster size. This means that for a given incident energy the plasmon mechanism of electron production will be efficient for relatively small nanoparticles, while the dipole plasmon mode will be suppressed for larger $D$. This behavior is illustrated in Figure 12. For instance, the number of low-energy electrons emitted via the surface plasmon mechanism from a 2 nm nanoparticle irradiated by a $0.3 \mathrm{MeV}$ proton (dashed red curve) is higher than that from the $4 \mathrm{~nm}$ nanoparticle (thin solid green curve). In the former case, the characteristic collision distance $v_{1} / \omega \approx 18$ a.u. becomes comparable with the nanoparticle radius, $D / 2=1 \mathrm{~nm} \approx 19$ a.u. Therefore, a larger nanoparticle with the diameter of $4 \mathrm{~nm}$ emits a smaller number of electrons via the plasmon mechanism. A small increase in the number of $5 \mathrm{eV}$ electrons produced by the $4 \mathrm{~nm}$ nanoparticle as compared to the smaller one is the result of an increased role of the volume plasmon due to the increased volume/surface ratio. A similar scenario holds for other incident velocities. For a $1 \mathrm{MeV}$ proton, the plasmon contribution to the electron yield saturates for the nanoparticle with the diameter of approximately $4 \mathrm{~nm}$, while for higher energies $\left(\varepsilon_{1}=10 \mathrm{MeV}\right)$ the saturation takes place for the $12 \mathrm{~nm}$ nanoparticle.

\section{CONCLUSION}

In this work, we have performed a detailed theoretical and numerical analysis of the electron production from gold nanoparticles due to irradiation by fast protons. It has been demonstrated that due to the prominent collective response to an external electric field, gold nanoparticles may significantly enhance the yield of low-energy secondary electrons in the medium. It has been shown that the significant increase in the number of emitted electrons comes from the two distinct types of collective electron excitations, namely plasmons and the excitation of $5 d$ electrons in individual atoms of a nanoparticle.

The analysis of the plasmon contribution has been performed within the model approach based on the plasmon resonance approximation. To justify parameters of the model, photoabsorption spectra of several gold nanoparticles have been calculated and compared with the spectra obtained by means of time-dependent density-functional theory. Our analysis has revealed that the broad feature positioned in the photoabsorption spectra above $20 \mathrm{eV}$ is related to the $5 d$ giant resonance 

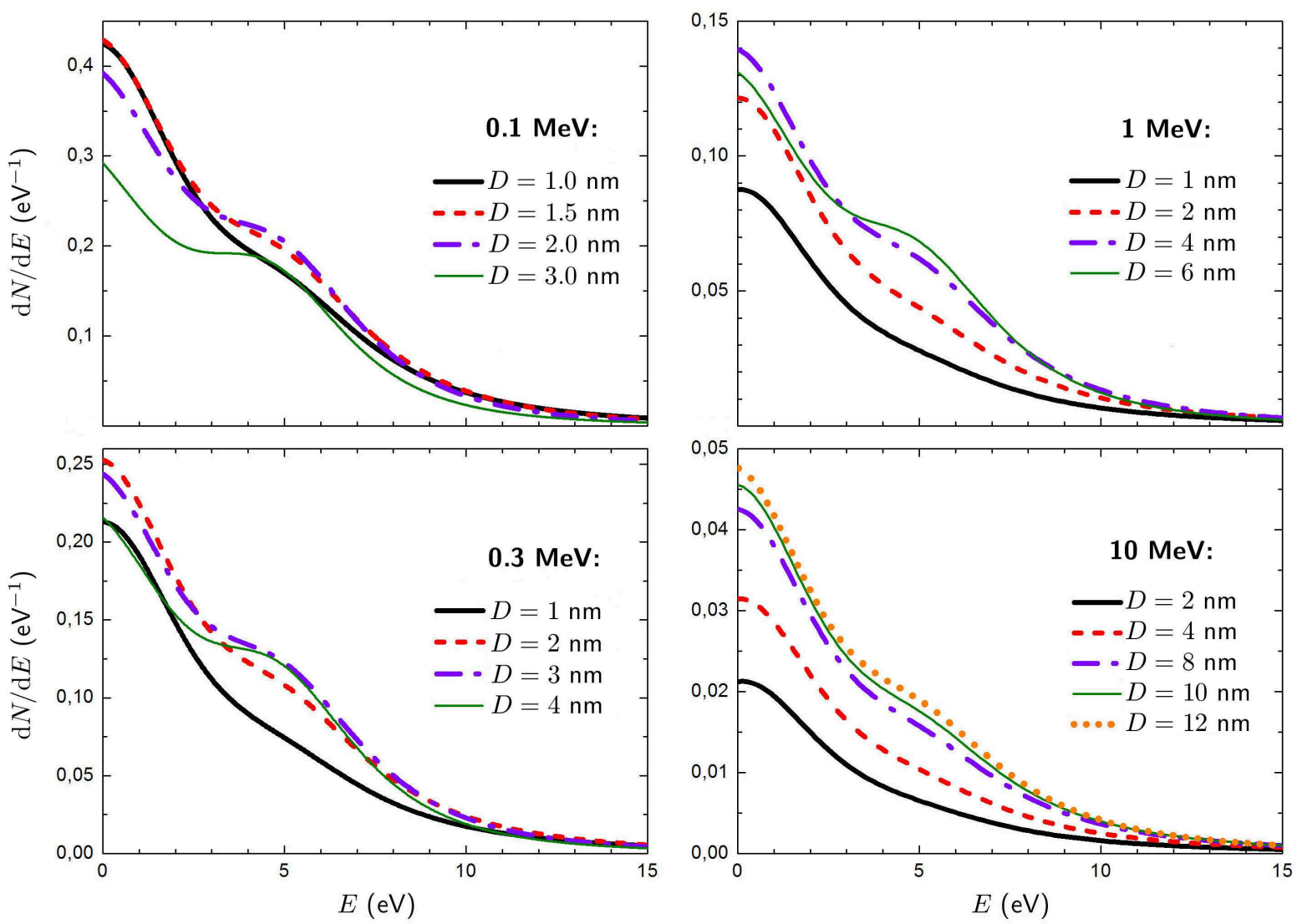

FIG. 12. Number of electrons per unit energy produced via the plasmon excitation mechanism in the solid gold nanoparticles of different size irradiated by the protons of different kinetic energy.

formed due to the excitation of electrons in the $5 d$ atomic shell. The low-energy peak below $10 \mathrm{eV}$ is caused by the formation of the surface plasmon, which arises due to collective excitation of delocalized outer-shell valence electrons in a whole cluster. The integration of the oscillator strength in this energy region has revealed that about 1.5 electrons from each atom contribute to this collective excitation.

The calculated yield of the electron production from gold nanoparticles has been compared to that from pure water medium, based on the dielectric formalism. It has been shown that the number of the low-energy electrons (with kinetic energy of about a few electronvolts) produced by the gold nanoparticle of a given size exceeds that produced by an equivalent volume of water by an order of magnitude. At the energies of about several $\mathrm{eV}$ and higher, there is a prominent contribution of the atomic $5 d$ electrons to the ionization cross section and, subsequently, to the electron production yield. Accounting for the collective electron excitations leads to a significant increase of the electron yield enhancement from the gold nanoparticle as compared to pure water. The importance of low-energy electrons is related to their ability to produce biodamage by dissociative electron attachment. The enhanced production of low-energy electrons will also lead to an increase in the number of free radicals as well as other reactive species, like hydrogen peroxide $\mathrm{H}_{2} \mathrm{O}_{2}$, which can travel the distances larger than the cell nucleus [10]. Thus, these species can deliver damaging impacts onto the DNA from the radiation induced damages associated with the presence of NPs in other cell compartments.

The utilized approach for evaluation of the electron production yield has an advantage as compared to other widely used approaches like track structure Monte Carlo simulations, which are utilized in the microdosimetric calculations (see Ref. [87] and references therein). In most of the Monte Carlo simulations, the contribution of collective excitations, which play a significant role in the ionization of gold and other noble metal nanoparticles, is not accounted for. An attempt to do it was made recently in Ref. [28], where the authors included the contribution of the volume plasmon excitation when calculating the cross sections of electron and proton impact on noble metal nanoparticles by means of Monte Carlo simulations. It was stated that the plasmon excitation does not play an important role in the process of electron emission from metallic nanoparticles, contributing much less to the overall cross sections than individual excitations. In this paper, we have demonstrated that the relative 
contribution of the surface collective electron excitation to the cross section exceeds that of the volume plasmon by an order of magnitude. Thus, the leading mechanism of electron production by gold nanoparticles is related to the surface plasmon. As a result of this analysis, we have demonstrated that the emission of low-energy electrons from the gold nanoparticles is a prominent effect, which should be accounted for when estimating the production of secondary electrons in a biological medium with embedded nanoparticles.

The calculated spectra of emitted electrons can further be used as the input data for the multiscale approach to the physics of radiation damage [11]. This approach has the goal of developing knowledge about biodamage at the nanoscale and molecular level and finding the relation between the characteristics of incident particles and the resultant damage $[11,15]$. Despite the fact that we have explored only the case of gold nanoparticles, we have introduced a general methodology, which can also be applied for other nanoscale systems, currently proposed as sensitizers in cancer therapy. The methodology can also be extended for collisions with heavier ions. In this case, the cross section obtained should be multiplied by a $Z^{2}$ scaling factor due to the change of Coulomb field of the projectile. Thus, the presented methodology can be utilized for studying irradiation of sensitizing nanoparticles by carbon ions, which are the most clinically used projectiles, besides protons.

\section{ACKNOWLEDGEMENTS}

We are grateful to Pablo de Vera for providing us the data on electron production in pure water medium. We also acknowledge the Frankfurt Center for Scientific Computing for the opportunity of carrying out resourcedemanding DFT calculations.
[1] K. Kobayashi, N. Usami, E. Porcel, S. Lacombe, and C. Le Sech, Mutation Res. 704, 123 (2010).

[2] D. M. Herold, I. J. Das, C. C. Stobbe, R. V. Iyer, and J. D. Chapman, Int. J. Radiat. Biol. 76, 1357 (2000).

[3] W. Chen and J. Zhang, J. Nanosci. Nanotechnol. 6, 1159 (2006).

[4] S. J. McMahon et al., Scientific Reports 1, 18 (2011); (Corrigendum: ibid. 3, 1725 (2013)).

[5] J. J. Hainfeld, D. N. Slatkin, and H. M. Smilowitz, Phys. Med. Biol. 49, N309 (2004).

[6] E. Porcel, S. Liehn, H. Remita, N. Usami, K. Kobayashi, Y. Furusawa, C. Le Sech, and S. Lacombe, Nanotechnology 21, 085103 (2010).

[7] J.-K. Kim, S.-J. Seo, K.-H. Kim, T.-J. Kim, M.-H. Chung, K.-R. Kim, and T.-K. Yang, Nanotechnology 21, 425102 (2010).

[8] C. Le Sech, K. Kobayashi, N. Usami, Y. Furusawa, E. Porcel, and S. Lacombe, Nanotechnology 23, 078001 (2012).

[9] P. Liu et al., Nanoscale 5, 11829 (2013).

[10] E. Porcel et al., Nanomed. Nanotech. Biol. Med. 10, 1601 (2014).

[11] E. Surdutovich and A. V. Solov'yov, Eur. Phys. J. D 68, 353 (2014).

[12] I. Bacarelli, F. A. Gianturco, E. Scifoni, A. V. Solov'yov, and E. Surdutovich, Eur. Phys. J. D 60, 1 (2010) (Topical issue on Molecular level assessments of radiation biodamage).

[13] D. Schardt, T. Elsässer and D. Schulz-Ertner, Rev. Mod. Phys. 82, 383 (2010).

[14] M. Durante and J. S. Loeffler, Nat. Rev. Clin. Oncol. 7, 37 (2010).

[15] A. V. Solov'yov, E. Surdutovich, E. Scifoni, I. Mishustin, and W. Greiner, Phys. Rev. E 79, 011909 (2009).

[16] B. D. Michael and P. O'Neill, Science 287, 1603 (2000).

[17] S. Denifl, T. D. Märk and P. Scheier, in Radiation Damage in Biomolecular Systems, edited by G. Garcia Gomez-Tejedor, M. C. Fuss (Springer Science+Business Media B.V., 2012), p. 45.
[18] M. A. Huels, B. Boudaiffa, P. Cloutier, D. Hunting, L. Sanche, J. Am. Chem. Soc. 125, 4467 (2003).

[19] B. Boudaïffa, P. Cloutier, D. Hunting, M. A. Huels, and L. Sanche, Science 287, 1658 (2000).

[20] M. Toulemonde, E. Surdutovich, and A. V. Solov'yov, Phys. Rev. E 80, 031913 (2009).

[21] X. Pan, P. Cloutier, D. Hunting, and L. Sanche, Phys. Rev. Lett. 90, 208102 (2003).

[22] U. Kreibig and M. Vollmer, Optical Properties of Metal Clusters (Springer-Verlag, Berlin-Heidelberg, 1995).

[23] P. M. Dinh, P.-G. Reinhard, and E. Suraud, An Introduction to Cluster Science (Wiley, 2013).

[24] C. Bréchignac, Ph. Cahuzac, F. Carlier, J. Leygnier, Chem. Phys. Lett. 164, 433 (1989).

[25] K. Selby, M. Vollmer, J. Masui, V. Kresin, W. A. de Heer, and W. D. Knight Phys. Rev. B. 40, 5417 (1989).

[26] Th. Fennel, K.-H. Meiwes-Broer, J. Tiggesbäumker, P.G. Reinhard, P. M. Dinh, and E. Suraud, Rev. Mod. Phys. 82, 1793 (2010).

[27] P. Wopperer, P. M. Dinh, P.-G. Reinhard, and E. Suraud, Phys. Rep. (2014), DOI: 10.1016/j.physrep.2014.07.003.

[28] C. Wälzlein, E. Scifoni, M. Krämer, and M. Durante, Phys. Med. Biol. 59, 1441 (2014).

[29] J.-P. Connerade, J. M. Esteva, and R. C. Karnatak Giant Resonances in Atoms, Molecules, and Solids (Plenum Press, New York, 1987).

[30] C. Bréchignac and J.-P. Connerade, J. Phys. B: At. Mol. Opt. Phys. 27, 3795 (1994).

[31] F. Xiao, Y. Zheng, P. Cloutier, Y. He, D. Hunting, and L. Sanche, Nanotechnology 22, 465101 (2011).

[32] Y. Zheng, D. J. Hunting, P. Ayotte, and L. Sanche, Radiat. Res. 169, 19 (2008); (Erratum: ibid. 169, 481 (2008))

[33] J.-P. Connerade and A. V. Solov'yov, Phys. Rev. A 66, 013207 (2002).

[34] A. V. Solov'yov, Int. J. Mod. Phys. B 19, 4143 (2005).

[35] A. V. Verkhovtsev, A. V. Korol, and A. V. Solov'yov, Eur. Phys. J. D 66, 253 (2012). 
[36] E. Runge and E. K. U. Gross, Phys. Rev. Lett. 52, 997 (1984).

[37] L. G. Gerchikov, A. N. Ipatov, and A. V. Solov'yov, J. Phys. B: At. Mol. Opt. Phys. 30, 5939 (1997).

[38] L. G. Gerchikov, A. N. Ipatov, R. G. Polozkov, and A. V. Solov'yov, Phys. Rev. A 62, 043201 (2000).

[39] A. V. Verkhovtsev, A. V. Korol, and A. V. Solov'yov, Phys. Rev. A 88, 043201 (2013).

[40] A. V. Verkhovtsev, A. V. Korol, and A. V. Solov'yov, J. Phys: Conf. Ser. 438, 012011 (2013).

[41] L. G. Gerchikov, A. V. Solov'yov, J.-P. Connerade, and W. Greiner, J. Phys. B: At. Mol. Opt. Phys. 30, 4133 (1997).

[42] L. G. Gerchikov, A. N. Ipatov, A. V. Solov'yov, and W. Greiner, J. Phys. B: At. Mol. Opt. Phys. 31, 3065 (1998).

[43] L. G. Gerchikov, P. V. Efimov, V. M. Mikoushkin, and A. V. Solov'yov, Phys. Rev. Lett. 81, 2707 (1998).

[44] A. V. Verkhovtsev, A. V. Korol, A. V. Solov'yov, P. Bolognesi, A. Ruocco, and L. Avaldi, J. Phys. B: At. Mol. Opt. Phys. 45141002 (2012).

[45] P. Bolognesi, A. Ruocco, L. Avaldi, A. V. Verkhovtsev, A. V. Korol, and A. V. Solov'yov, Eur. Phys. J. D 66, 254 (2012).

[46] J.-P. Connerade and A. V. Solov'yov, J. Phys. B: At. Mol. Opt. Phys. 29, 365 (1996).

[47] L. G. Gerchikov, A. N. Ipatov, and A. V. Solov'yov, J. Phys. B: At. Mol. Opt. Phys. 31, 2331 (1998).

[48] A. V. Korol and A. V. Solov'yov, Polarization Bremsstrahlung, Springer Series on Atomic, Optical, and Plasma Physics, Vol. 80 (Springer Berlin Heidelberg, 2014).

[49] S. Bulusu, X. Li, L. S. Wang, and X. C. Zeng, Proc. Natl. Acad. Sci. 103, 8326 (2006).

[50] J. Li, X. Li, H.-J. Zhai, and L.-S. Wang, Science 299, 864 (2003).

[51] M. P. Johansson, D. Sundholm, and J. Vaara, Angew. Chem. Int. Ed. 43, 2678 (2004).

[52] X. Gu, M. Ji, S. H. Wei, and X. G. Gong, Phys. Rev. B 70, 205401 (2004).

[53] Y. Gao and X. C. Zeng, J. Am. Chem. Soc. 127, 3698 (2005).

[54] M. J. Frisch et al., Gaussian 09 Revision A.1, Gaussian Inc. Wallingford CT, 2009.

[55] W. J. Stevens, M. Krauss, H. Basch, and P. G. Jasien, Can. J. Chem. 70, 612 (1992).

[56] J. P. Perdew, K. Burke, and M. Ernzerhof, Phys. Rev. Lett. 77, 3865 (1996).

[57] R. Kubo, J. Phys. Soc. Jpn. 12, 570 (1957).

[58] B. Walker, A. M. Saitta, R. Gebauer, and S. Baroni, Phys. Rev. Lett. 96, 113001 (2006).

[59] B. Walker and R. Gebauer, J. Chem. Phys. 127, 164106 (2007).

[60] D. Rocca, R. Gebauer, Y. Saad, and S. Baroni, J. Chem. Phys. 128, 154105 (2008).

[61] O. B. Malcioğlu, R. Gebauer, D. Rocca, and S. Baroni, Comp. Phys. Commun. 182, 1744 (2011).
[62] P. Giannozzi et al., J. Phys.: Condens. Matter 21, 395502 (2009).

[63] D. Vanderbilt, Phys. Rev. B 41, 7892(R) (1990).

[64] Ph. Lambin, A. A. Lucas, and J.-P. Vigneron, Phys. Rev. B 46, 1794 (1992).

[65] D. Östling, P. Apell, and A. Rosen, Europhys. Lett. 21, 539 (1993).

[66] S. Lo, A. V. Korol, and A. V. Solov'yov, J. Phys. B: At. Mol. Opt. Phys. 40, 3973 (2007).

[67] A. V. Korol and A. V. Solov'yov, Phys. Rev. Lett. 98, 179601 (2007).

[68] D. I. Palade and V. Baran, Optical response of $C_{60}$ fullerene from a Time Dependent Thomas Fermi approach, arXiv:1408.1899 [physics.atm-clus].

[69] B. L. Henke, E. M. Gullikson, and J. C. Davis, At. Data Nucl. Data Tables 54, 181 (1993).

[70] U. Fano, Phys. Rev. 124, 1866 (1961).

[71] U. Fano, Rev. Mod. Phys. 64, 313 (1992).

[72] S. Malola, L. Lehtovaara, J. Enkovaara, and H. Häkkinen, ACS Nano 7, 10263 (2013).

[73] N. Durante, A. Fortunelli, M. Broyer, and M. Stener, J. Phys. Chem. C 115, 6277 (2011).

[74] R. Philip, P. Chantharasupawong, H. Qian, R. Jin, and J. Thomas, Nano Lett. 12, 4661 (2012).

[75] M. Stener, A. Nardelli, R. De Francesco, and G. Fronzoni, J. Phys. Chem. C 111, 11862 (2007).

[76] M. S. Dresselhaus, G. Dresselhaus, and A. Jorio, Group Theory: Application to the Physics of Condensed Matter (Springer, 2010).

[77] C. M. Aikens, S. Li, and G. C. Schatz, J. Phys. Chem. C 112, 11272 (2008).

[78] E. M. Fernández, J. M. Soler, and L. C. Balbás, Phys. Rev. B 73, 235433 (2006).

[79] L. G. Gerchikov, A. N. Ipatov, A. V. Solov'yov, and W. Greiner, J. Phys. B: At. Mol. Opt. Phys. 33, 4905 (2000).

[80] E. Scifoni, E. Surdutovich, and A. V. Solov'yov, Phys. Rev. E 81, 021903 (2010).

[81] P. de Vera, R. Garcia-Molina, I. Abril, and A. V. Solov'yov, Phys. Rev. Lett. 110, 184104 (2013).

[82] G. B. Sushko, V. G. Bezchastnov, I. A. Solov'yov, A. V. Korol, W. Greiner, and A. V. Solov'yov, J. Comput. Phys. 252, 404 (2013).

[83] I. A. Solov'yov, A. V. Yakubovich, P. V. Nikolaev, I. Volkovets, and A. V. Solov'yov, J. Comput. Chem. 33, 2412 (2012).

[84] L. D. Landau and E. M. Lifshitz, Quantum Mechanics: Non-Relativistic Theory (3rd ed.), Course of Theoretical Physics, Vol. 3. (Butterworth-Heinemann, 1976).

[85] E. Surdutovich, O. I. Obolensky, E. Scifoni, I. Pshenichnov, I. Mishustin, A. V. Solov'yov, and W. Greiner, Eur. Phys. J. D 51, 63 (2007).

[86] E. Surdutovich and A. V. Solov'yov, Phys. Rev. E 82, 051915 (2010).

[87] C. Wälzlein, Nanometer Scale Description of Electron Transport and Damage in Condensed Media Using the TRAX Monte Carlo Code. Ph.D. thesis. Fachbereich Physik, Technische Universität Darmstadt (2014). 